\title{
On the Zero- and First-Order Interpolation in Synthesized Sine Waves for Testing Purposes
}

\author{
Albert Lozano, Student Member, IEEE, Javier Rosell, Member, IEEE, and \\ Ramón Pallás-Areny, Senior Member, IEEE
}

\begin{abstract}
This paper deals with the quality requirements of synthesized sine waves reconstructed through a zero- or firstorder hold for testing purposes, in particular when a switching demodulator is used. In this case, the interest is to have great spectral purity near the carrier rather than in all of the band. We show that the best method to obtain great spectral purity in the frequency band close to the synthesized frequency is by using zero-order hold instead of a first-order hold. Results show that even though a first-order hold implies a decrease of total harmonic amplitude distortion (THD), the measured spurious harmonics are kept lower or equal when using a zero-order hold in the 15 closest components. We conclude that in testing applications a zero-order hold (in spite of its greater THD) yields better results.
\end{abstract}

\section{INTRODUCTION}

CINE waves are frequently used for testing and characterization purposes in electronic instrumentation. Some examples are: measurement of audio frequency amplifiers [1], calibration and testing of wattmeters with well-known distorted waveforms [2], searching for nonlinearities in passive electrical components [3], and invivo characterization of biological tissues [4], [5] by measuring the frequency dependence of their impedance. Some of the requirements of sine wave generators intended for these applications are:

- provision for easy change of frequency;

- capability of generating complex waveforms, for example, the sum of two or more sine waves; in some applications such as body fluid shift monitoring, the desired information relies on the ratio of body impedance at low and high frequency, rather than on the absolute value of the impedance [6];

- simultaneous generation of control signals to drive a coherent (synchronous) detector to detect both inphase and quadrature components of the tested system output signal;

Manuscript received May 14, 1992; revised August 26, 1992. This work has been supported by the Dirección General de Investigación Científica y Técnica, Ministerio de Educación y Ciencia, Project PB 89-0505.

The authors are with the Divisió d'Instrumentació i Bioenginyeria, Departament d'Enginyeria Electrònica-UPC, Barcelona, Spain.

IEEE Log Number 92047620.
- the possibility of phase-shift adjustment in control signals in order to compensate for phase shifts introduced by electronic circuitry, which can be even larger than the one intended to be measured;

- the generation of a waveform with low distortion.

A common requirement in the above measurement situations is the need to obtain an injection signal with great spectral purity in the nearby frequency band rather than in a broad band. The basic concepts on the reconstruction of sampled signals with a zero- and first-order hold can be found, for example, in [7]. In this paper we show that to obtain a great spectral purity in the frequency band close to the synthesized frequency it is better to use a staircase waveform or zero-order hold instead of a first-order hold or linear interpolation between the signal's samples.

\section{Harmonic Prediction in Zero-Order and First- ORdER INTERPOLATION}

An attractive approach to satisfy the above requirements for sine wave generators intended for measurement purposes is to use a digital synthesizer [8], [9]. This consists of the sequential reading of data stored in a solid state memory, which is the digitized version of the mathematical equation for the desired waveform, and its further reconstruction by a digital-to-analogue converter (DAC). The basics of this technique, also named Direct Digital Synthesis, have been used in communication systems [9] and together with other techniques that allow extending the frequency band to RF range [10].

A zero-order sample reconstruction, that is to take directly the date from the DAC output, gives a staircase waveform with a ripple that depends on the number of samples used to reconstruct the signal. The staircase waveform frequency spectrum is [11]

$$
H(f)=\frac{1}{2} \sum_{m=-\infty}^{+\infty} \operatorname{sinc}\left(\frac{m}{N}\right) \delta\left(f-m f_{0}\right)
$$

where

$$
\operatorname{sinc}(x)=\frac{\sin (\pi x)}{\pi x},
$$




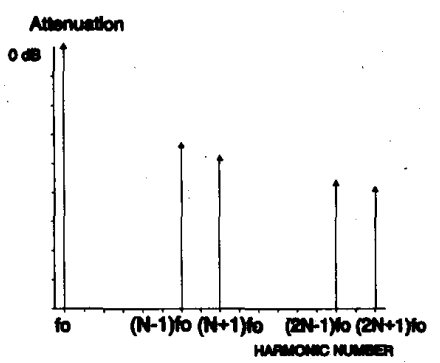

Fig. 1. Ideal frequency spectrum of a synthesized sine wave.

$m=(k N \pm 1)$,

$k$ is any integer,

$N$ is the number of samples used to reconstruct the signal, and $f_{0}$ is the generated sine wave frequency.

Therefore, as it can be seen in Fig. 1, in addition to the fundamental frequency $f_{o}$, some other frequency components appear, separated $(k N \pm 1) f_{o}$ from it. We shall call these components "predicted harmonics."

The amplitude of the $j$ th harmonic component can be written as

$$
\left|a_{j}\right|=\frac{1}{2} \operatorname{sinc}\left(\frac{j}{N}\right) .
$$

The respective amplitude of these undesired harmonics relative to that of the fundamental $\left(a_{1}\right)$ is called attenuation $A_{j z o}$ (where ${ }_{z o}$ stands for the zero-order interpolation). Its value in decibels is

$$
\begin{aligned}
& A_{j z o}(d B)=20 \log \frac{\left|a_{1}\right|}{\left|a_{j}\right|} \\
& A_{j z o}(d B)=20 \log \frac{\operatorname{sinc}\left(\frac{1}{N}\right)}{\operatorname{sinc}\left(\frac{j}{N}\right)}
\end{aligned}
$$

so that

$$
A_{j z o}(d B)=20 \log (j) \frac{\sin \left(\frac{\pi}{N}\right)}{\sin \left(\frac{j \pi}{N}\right)}
$$

and substituting $j=(k N \pm 1)$ we obtain

$$
A_{j z o}(d B)=20 \log (k N \pm 1) \text {. }
$$

In order to increase the attenuation for the undesired spectral lines, some other approaches have been proposed. A common approach consists of using a linear interpolation or first-order hold instead of a zero-onder bold [11], [12]. Linear interpolation can be implemented by using two memories, two DAC's and adding their outputs, as shown in Fig. 2. The firat memory contains the same information as was stored to produce the staircase waveform, while the second memory contains the information for generating the chords in each staircase step.

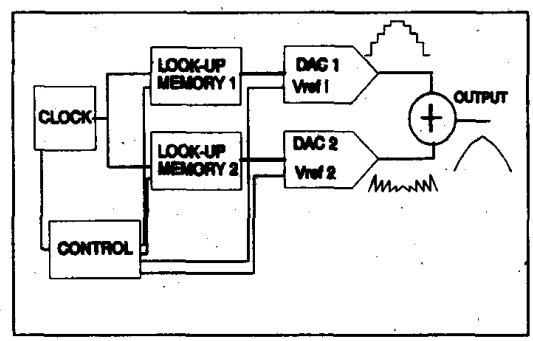

Fig. 2. Block diagram of a linear interpolation approach.

Therefore when using this method, the voltage reference for the second DAC cannot be a continuous signal; it must be a sawtooth signal. Whatever the circuit to implement it, linear interpolation not only implies increasing the number of components, but it also requires additional control logic. Additional techniques for implementing linear interpolation are described in [12], but all of them need the use of two memories and to manipulate the reference voltage signals to the DAC.

When using a first-order hold, the amplitude of the $j$ th harmonic component is [11]

$$
\left|a_{j}\right|=\frac{1}{2} \operatorname{sinc}^{2}\left(\frac{j}{N}\right)
$$

and the respective attenuation, $A_{j f o}$, ( $f_{o}$ standing first-order hold case) is calculated to be

$$
A_{j f o}(d B)=40 \log (k N \pm 1) .
$$

\section{Synthesizer Performance: Experimental and Simulation Results}

The relative performance of each interpolator with respect to harmonic generation can be evaluated by comparing (4) and (6). From these equations, it is obvious that total harmonic distortion (THD) is considerably smaller in the case of linear interpolation than in the staircase waveform. However, even though THD has been traditionally used to specify the purity of a waveform, it is not always the most suitable parameter.

First of all, it is important to notice that unpredicted harmonic components not given by (1) will appear in practice [11], [12]. These unpredicted harmonic components are attributed to the finite word length of data stored in the look-up memory, to the finite precision of the DAC and to differences in delays among its input digital liaes. Furthermore, when using linear interpolation, unpredicted harmonics increase because of the contributions from the additional hardware, for example, channel mismatches, offsets and drifts in voltage references if two DACs are used. The generated harmonic components different from the fundamental one will be a source of errors when a switching demodulator is used for voltage detection [13]. In this case the most harmful harmonic components will be those close to the carrier, so it is essential for them to be as attenuated as possible. 
Secondly, when the synthesizer generates the sum of two sine waves it is extremely important for the band between the low-frequency signal $\left(f_{1}\right)$ and the high-frequency signal $\left(f_{2}\right)$ to be as clear as possible. Harmonic components far from the higher frequency can be easily removed by low-pass filtering, while the suppression of harmonics between $f_{1}$ and $f_{2}$ would become more difficult. Fig. 3 shows the actual frequency spectrum for this case. Here, it is essential that the band, between both synthesized sine waves is as clear as possible. Therefore, the number of samples must be chosen so that the frequency of the first predicted harmonic - that will be placed at $((N$ - 1) $f_{1}$ )-is higher than $f_{2}$. From these considerations we conclude that a better performance criterion for synthesized sine waves is the attenuation of harmonics near the carrier, rather than in the whole band as measured by THD.

By using a spectrum analyzer, we have measured the harmonic attenuation in the case of a zero-hold, and compared it with that given in [11] for linear interpolation. In this experiment we have used a data length of 8 bits, 32 samples per period in the sampled signal, and a DAC with a nonlinearity error less than $0.19 \%$. The result is displayed in Table I. Even though linear interpolation results in lower THD as expected from (4) and (6), the amplitude of the unpredicted harmonics is larger than when using a zero-order hold.

The amplitude of the harmonics can be decreased by increasing the number of bits used for each sample, and by increasing the number of samples used to reconstruct the signal. We have carried out computer simulations consisting of sampling the sine wave, truncating it depending on the number of bits used to store a sample, and analyzing the resulting spectrum when the word length or the number of samples per cycle is changed. The equation we have used to obtain the harmonic amplitude spectrum in both cases, as well as the first-order hold simulation, is the same as described in [12]. When the word length is increased we have found through these computer simulations that the amplitude of unpredicted harmonics is lower, but without any marked trend; for example, an increase from 8 to 10 bits implies a nonuniform decrease in the fifth, seventh, and ninth harmonic level, whereas the eleventh one keeps at the same level, as shown in Fig. 4. This effect is highlighted in the high-order harmonic components. On the other hand, the level of predicted harmonics remains the same, in accordance with (4) or (6), regardless of word length.

For the zero-order hold case, when the number of samples $N$ is increased we have measured a decrease in the amplitude of both predicted and unpredicted harmonic components. The predicted harmonics decrease in accordance with (4), as do the unpredicted harmonics, but these follow a more erratic trend. Furthermore, for increasing $N$, the predicted harmonics not only decrease their amplitude but also move far away from the fundamental frequency, thus facilitating elimination by filtering. However, increasing the number of samples forces us to

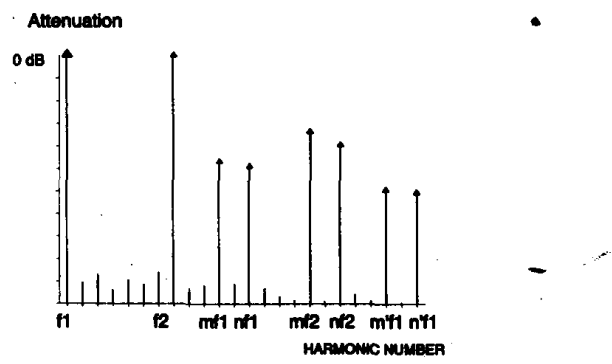

Fig. 3. Real frequency spectrum obtained when the sum of two sine waves is reconstructed by means of synthesis techniques.

TABLE I

\begin{tabular}{ccc}
\hline $\boldsymbol{n}$ & Zero-order & First-order \\
\hline 3 & 58 & 50 \\
5 & 60 & 60 \\
7 & 58 & 58 \\
9 & 62 & 58 \\
11 & 74 & 78 \\
13 & 62 & 58 \\
15 & 60 & 60 \\
17 & 72 & 70 \\
19 & 64 & 62 \\
21 & 84 & 84 \\
23 & 80 & 72 \\
35 & 81 & 74 \\
37 & 84 & 84 \\
29 & 86 & 86 \\
31 & 28 & 54 \\
33 & 28 & 58 \\
\hline
\end{tabular}

Attenuation levels referreed to fundamental frequency for unpredicted harmonics of a sine wave synthesized using a zero-order interpolator and a first-order interpolator. Data length is 8 bits; $N$ is 32 samples/period.

increase the frequency at which the memory is read in order to maintain the output frequency constant, and the maximum reading frequency is limited by the minimum memory access time.

Fig. 4 shows the levels of predicted and unpredicted harmonic components in synthesized sine waves obtained by simulation for zero- and first-order hold with word lengths of 8 and 10 bits. It is important to notice that a first-order hold does not lead to a decrease in harmonic amplitude until the 15th. Harmonic amplitude is increased in practice by hardware limitations which are more significant in the interpolated waveforms. We have also noticed that there is no appreciable difference in either case in the level of the most harmful unpredicted harmonics, provided that the number of samples used is greater than 32 per cycle and word length is 8 or more bits.

\section{CONCLUSION}

We have analyzed the effects of using a staircase waveform (zero-order hold) or a linear interpolated waveform (first-order hold) to synthesize a sine wave. From experimental results we conclude that in testing applications requiring great spectral purity near the desired frequency, the staircase waveform yields better results than a linearly interpolated waveform. 


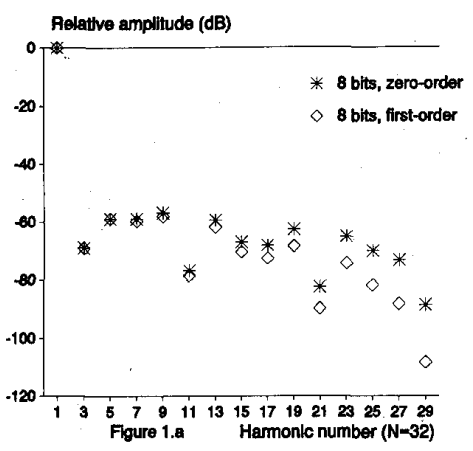

(a)

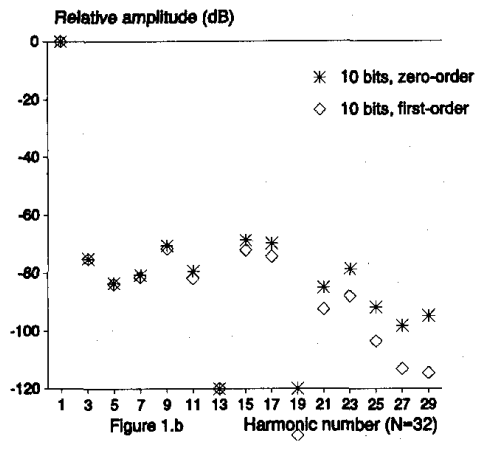

(b)

Fig. 4. (a) Simulated attenuation levels using 8 bits per sample and 32 samples/period to reconstruct a sine wave. (b) Simulated attenuation levels using 10 bits per sample and 32 samples/period to reconstruct a sine wave.

The linearly interpolated waveform considerably reduces the signal THD, but it has harmonic components near the carrier which are larger than those of a staircase waveform. This indicates that THD is not always the best performance criterion when generating testing waveforms.

From computer simulations we have inferred that two possible ways of reducing the harmonic component amplitude are to increase the number of samples per period used to store the digitized signal and to increase the bit length of the word used to store a sample. Nevertheless; the improvement is limited in practice by shortcomings of practical circuits, which are more significant in a firstorder hold circuit, thus rendering it less suitable than a zero-order hold.

\section{REFERENCES}

[1] G. W. Small and K. E. Leslie, "Synthesizing signal generator for use with lock-in amplifiers in audiofrequency measurements," IEEE Trans. Instrum. Meas., vol. IM-35, pp. 249-255, Sept. 1986.

[2] H. Ahlers, L. Rahf, H. Hartwig, and J. D. Sievert, "Programmable digital two-channel function generator for testing power meters," IEEE Trans. Instrum. Meas., vol. IM-34, pp. 231-234, June 1985.

[3] M. Kanno and I. Minowa, "Application of nonlinearity measuring method using two frequencies to electrical components," IEEE Trans. Instrum. Meas., vol. IM-34, pp. 590-593, Dec. 1985.

[4] L. E. Baker, "Principles of the impedance technique," IEEE Eng. in Med and Biol. Magazine, vol. 8, no. 1, pp. 11-15, March 1989.

[5] B. H. Brown, D. C. Barber, and L. Tarasenko, "Electrical impedance tomography-applied potential tomography," Clin. Phys. Physiol. Meas., vol. 8, Suppl. A, 1987.

[6] H. G. Goovaerts, F. R. de Vries, J. H. Meijer, P. M. J. M. De vries, A. J. M. Donker, and H. Schreider, "Microprocessor-based system for measurement of electrical impedances during haemodialysis and in postoperative care," Med. \& Biol. Eng. \& Comput., vol. 26, pp. 75-80, Jan. 1988.

[7] B. C. Kuo, Analysis and Synthesis of Sampled-Data Control Systems. Englewood Cliffs, NJ: Prentice-Hall, 1963.

[8] D. D. Caviglia, A. De Gloria, G. Donzellini, G. Parodi, and D. Porta, "Design and construction of an arbitrary waveform generator," IEEE Trans. Instrum. Meas., vol. IM-32, pp. 398-403, Sept. 1983.

[9] E. McCune, "Quantitative prediction of DDS Spurs," Digital RF Sol. Corp., AN1012, 1991.

[10] "Hybryd PLL/DDS Frequency Synthesizers," Qualcomm Inc., Application Note CL80-3459-1.

[11] W. A. Evans and M. S. Towers, "Hybrid technique in waveform generation and synthesis," IEEE Proc., vol. 127, Pt.G, pp. 119-128, June 1980.

[12] M. S. Towers, "Programmable waveform generator using linear interpolation with multiplying D/A convertors," IEEE Proc., vol. 129 Pt.G, pp. 19-25, Feb. 1982.

[13] M. L. Meade, Lock-In Amplifiers: Principles and Applications (IEE Electrical Measurement Series 1). London: Peter Peregrinus Ltd., 1983. 